\title{
Exciton-Exciton Annihilation in Two-Dimensional Halide Perovskites at Room Temperature
}

\author{
Géraud Delport ${ }^{1}$, Gabriel Chehade ${ }^{1}$, Ferdinand Lédée ${ }^{1}$, Hiba Diab ${ }^{1}$, Cosme Milesi-Brault $^{1}$, \\ Gaëlle Trippé-Allard ${ }^{1}$, Jacky Even ${ }^{2}$, Jean-Sébastien Lauret ${ }^{1}$, Emmanuelle Deleporte $^{1}$ and Damien Garrot ${ }^{3 a}$ \\ 1) Laboratoire Aimé Cotton, CNRS, Univ. Paris-Sud, ENS Paris-Saclay, Université Paris-Saclay, \\ 91405 Orsay Cedex \\ 2) Univ Rennes, INSA Rennes, CNRS, Institut FOTON - UMR 6082, Rennes F-35000, France \\ 3) Groupe d'Etude de la Matière Condensée, CNRS, Université de Versailles Saint-Quentin-En- \\ Yvelines, Université Paris-Saclay, 45 Avenue des Etats-Unis, 78035, Versailles, France
}

a. Corresponding author: damien.garrot@uvsq.fr

\section{Supporting Information}

\section{Experimental Methods}

Crystals synthesis Crystals have been synthesized following the "Anti-solvent Vapor-assisted Capping Crystallization" method for $\mathrm{n}=1$. $^{1}$ The process has been modified to grow pure phase crystals of $\left(\mathrm{C}_{6} \mathrm{H}_{5} \mathrm{C}_{2} \mathrm{H}_{4} \mathrm{NH}_{3}\right)_{2}\left(\mathrm{CH}_{3} \mathrm{NH}_{3}\right)_{n-1} \mathrm{~Pb}_{n} \mathrm{I}_{3 \mathrm{n}+1}$ with $\mathrm{n}=2$ and $\mathrm{n}=4$. The details of the synthesis will be published elsewhere. RPPs with $\mathrm{n}=3$ have been synthesized following the slow cooling method reported by Peng et al.. $744 \mathrm{mg}$ of lead (II) oxyde PbO, $1272 \mathrm{mg}$ of methylammonium iodide MAl and $82 \mathrm{mg}$ of phenylethylammonium iodide PEAl were dissolved in $10 \mathrm{~mL}$ hydriodic acid solution (stabilized, $1.5 \% \mathrm{w}$ $\mathrm{H}_{3} \mathrm{PO}_{2}$, Sigma-Aldrich). The precursors ratio is $3.33 / 8 / 0.33$. The solution was stirred at $90^{\circ} \mathrm{C}$ for a dozen of minutes resulting in a clear pale yellow solution. The stirring was subsequently discontinued, and the solution was left to cool to room temperature. The square-shaped, black crystals were then isolated by suction filtration and dried under vacuum at room temperature.

Optical Spectroscopy PL spectra were detected with a Pixis 100B CCD camera (Ropers Scientific) mounted on a Spectrapro 2500i spectrometer. For Time-Resolved Photoluminescence (TRPL), the excitation source was a pico-second laser diode at $405 \mathrm{~nm}$ (PicoQuant). The PL decays were measured with the timecorrelated single-photon counting TimeHarp 260 system from PicoQuant. The Instrumental Response Function (IRF) of the TRPL set-up is displayed in Figure S1. 


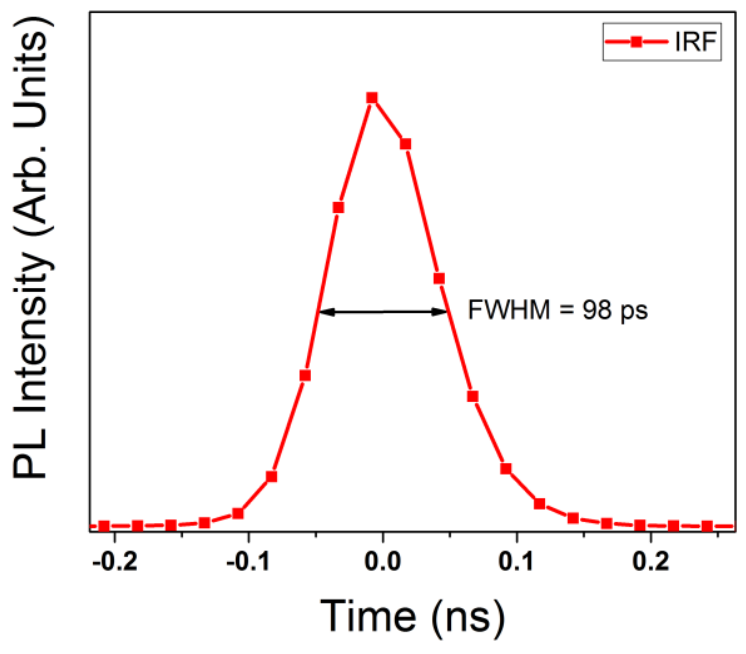

Figure S1: Instrumental Response Function (IRF) of the TRPL set-up.

\section{Carrier density calculation}

The carrier density has been estimated using an absorption coefficient of $1.0 \times 10^{5} \mathrm{~cm}^{-1}$ extracted from transmission measurement on $n=1$ pure phase thin films. ${ }^{3}$ We consider the photoexcitation of carriers on a depth corresponding to the penetration length of the material $(1 / \alpha)$ and take the average on the number of perovskite layers. The refractive index was estimated from ellipsometry measurements and the reflectance $R$ was calculated from Fresnel equation and estimated to $15 \%$ at $405 \mathrm{~nm}$. The carrier density has been estimated using the equation: $(1-R)(1-T) P /\left(h v \cdot \pi r^{2} N\right)$ where $\mathrm{T}$ is the transmittance, $\mathrm{P}$ is the excitation power, $h$ the Planck's constant, $r$ is the size of the excitation spot, $\alpha$ is the absorption coefficient and $\mathrm{N}$ is the number of perovskite layers.

\section{Exciton binding energy}

Blancon et al. have proposed a general scaling law of the exciton binding energies with the perovskite layer thickness:

$$
E_{b, 1 s}=\frac{E_{0}}{\left(1+\frac{\propto-3}{2}\right)^{2}}
$$

With $\alpha=3-\gamma e^{-\frac{L_{W}}{2 a_{0}}}$

Where $E_{0}$ and $a_{0}$ are the 3D Rydberg energy and Bohr radius of 3D perovskites respectively, $L_{w}$ is the width of the perovskite layer and $\gamma$ is a correction factor due to dielectric confinement effects. ${ }^{4} \mathrm{~A}$ factor $\gamma=1.76$ was reported for BEA based RPPs. Here, we find a lower value of $\gamma=1.56$, which is consistent with the lower dielectric contrast between the organic and inorganic part in PEA based perovskites. ${ }^{5}$ 


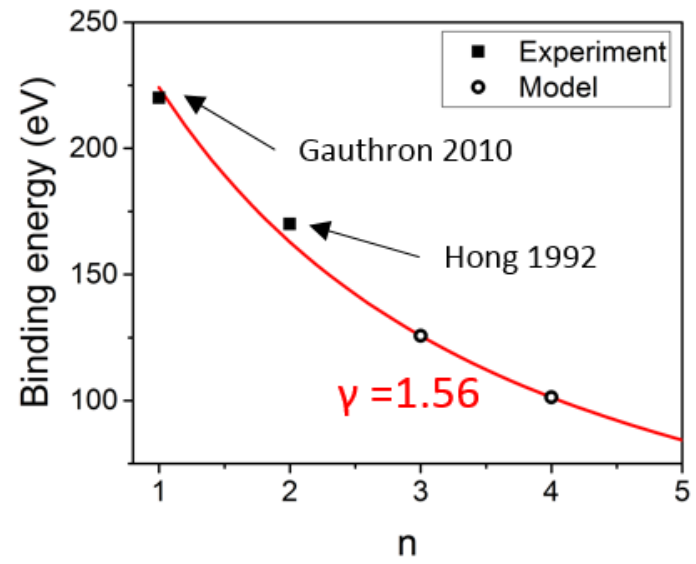

Figure S2: Exciton binding energy as function of the $n$ parameter for $\left(\mathrm{C}_{6} \mathrm{H}_{5} \mathrm{C}_{2} \mathrm{H}_{4} \mathrm{NH}_{3}\right)_{2}\left(\mathrm{CH}_{3} \mathrm{NH}_{3}\right)_{n-1} \mathrm{~Pb}_{n} \mathrm{I}_{3 n+1}$, black squares are experimental values from ref. ${ }^{5,6}$. In red, general scaling law from ref. ${ }^{4}$ with $L_{w}=0.6 \times n$ in nanometers, $\gamma=1.56, E_{0}=16 \mathrm{meV}, a_{0}=4.6 \mathrm{~nm}$. Open circles show the binding energy estimated from the scaling law.

a)
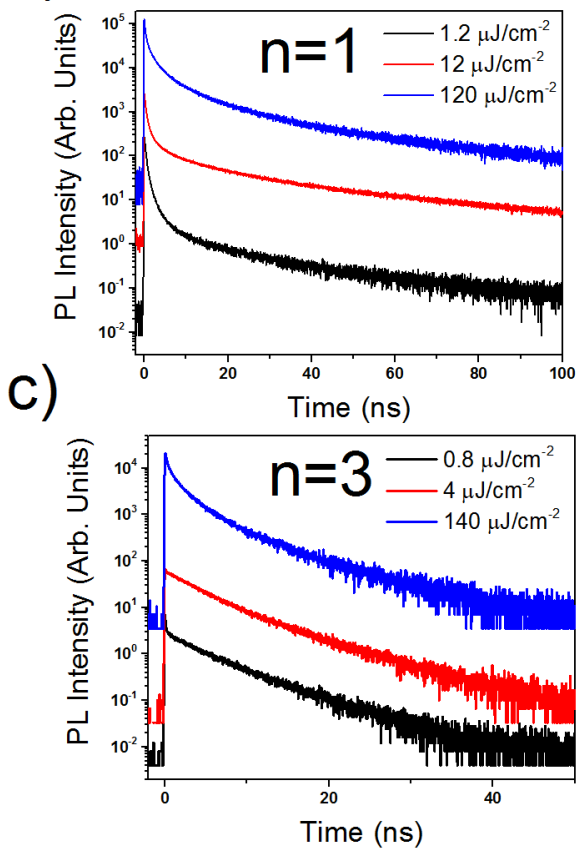

b)
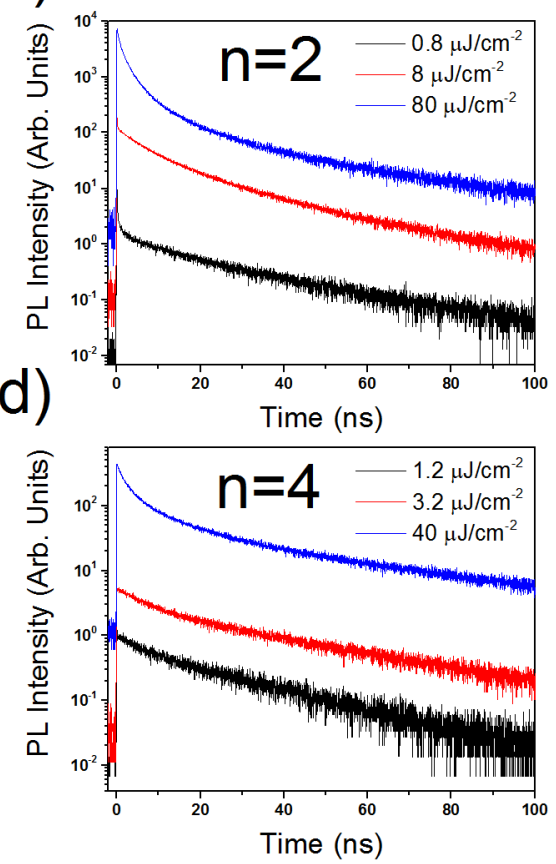

Figure S3: Evolution of the TRPL response for different power excitations for a) $n=1$ b) $n=2$ c) $n=3$ and d) n=4 RPP crystals. 


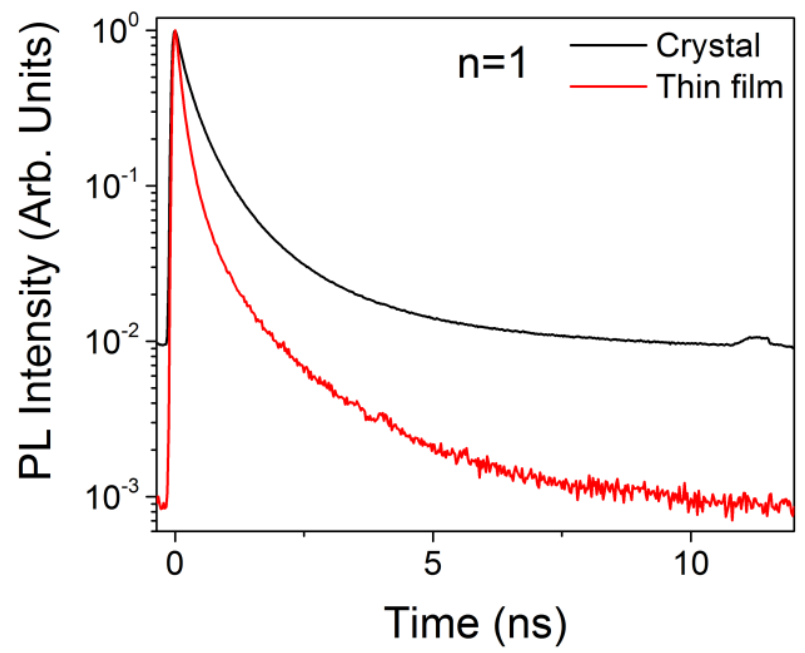

Figure S4: TRPL dynamics measured on a crystal and on a thin film of $\left(\mathrm{C}_{6} \mathrm{H}_{5} \mathrm{C}_{2} \mathrm{H}_{4} \mathrm{NH}_{3}\right)_{2} \mathrm{Pbl}_{4}(n=1)$.

\section{Average lifetimes}

In order to compare the evolution of the lifetime as function of excitation power and $\mathrm{n}$ value, the PL decays have also been fitted with a sum of exponential: $I(t)=\sum_{i} A_{i} e^{-t / \tau_{i}}$, where $\mathrm{A}_{\mathrm{i}}$ is the amplitude and $\tau_{\mathrm{i}}$ the characteristic lifetime of the exponential. The evolution of the average lifetimes, defined as $\langle\tau\rangle=$ $\sum_{i} A_{i} \tau_{i} / \sum_{i} A_{i}$ are reported in Figure $\mathrm{S} 5$.

a) Carrier density $\left(\mathrm{cm}^{-2}\right) \quad$ b

b) Carrier density $\left(\mathrm{cm}^{-2}\right)$
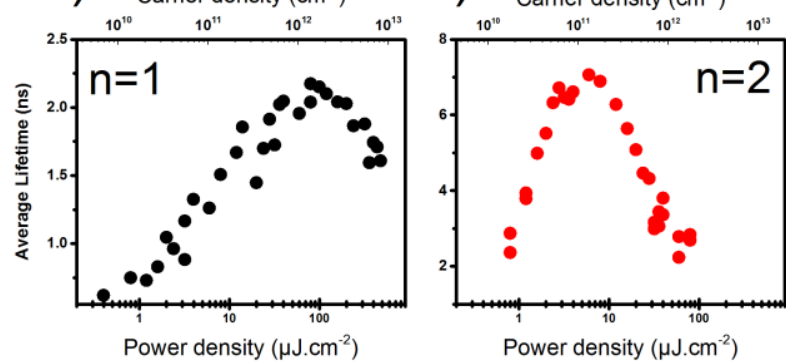

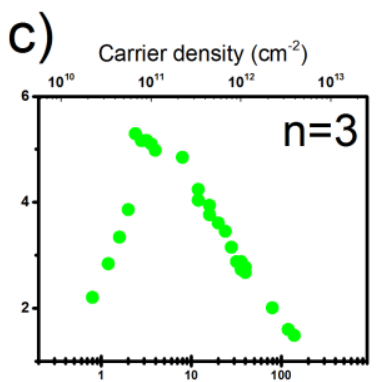

Power density $\left(\mu \mathrm{J} . \mathrm{cm}^{-2}\right)$ d) Carrier density $\left(\mathrm{cm}^{-2}\right)$

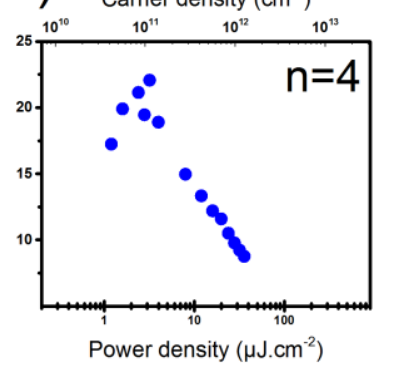

Figure S5: Evolution of the average lifetimes as function of power excitation for a) $n=1$ b) $n=2$ c) $n=3$ d) $n=4$. Top $x$-axis, estimated carrier density. 

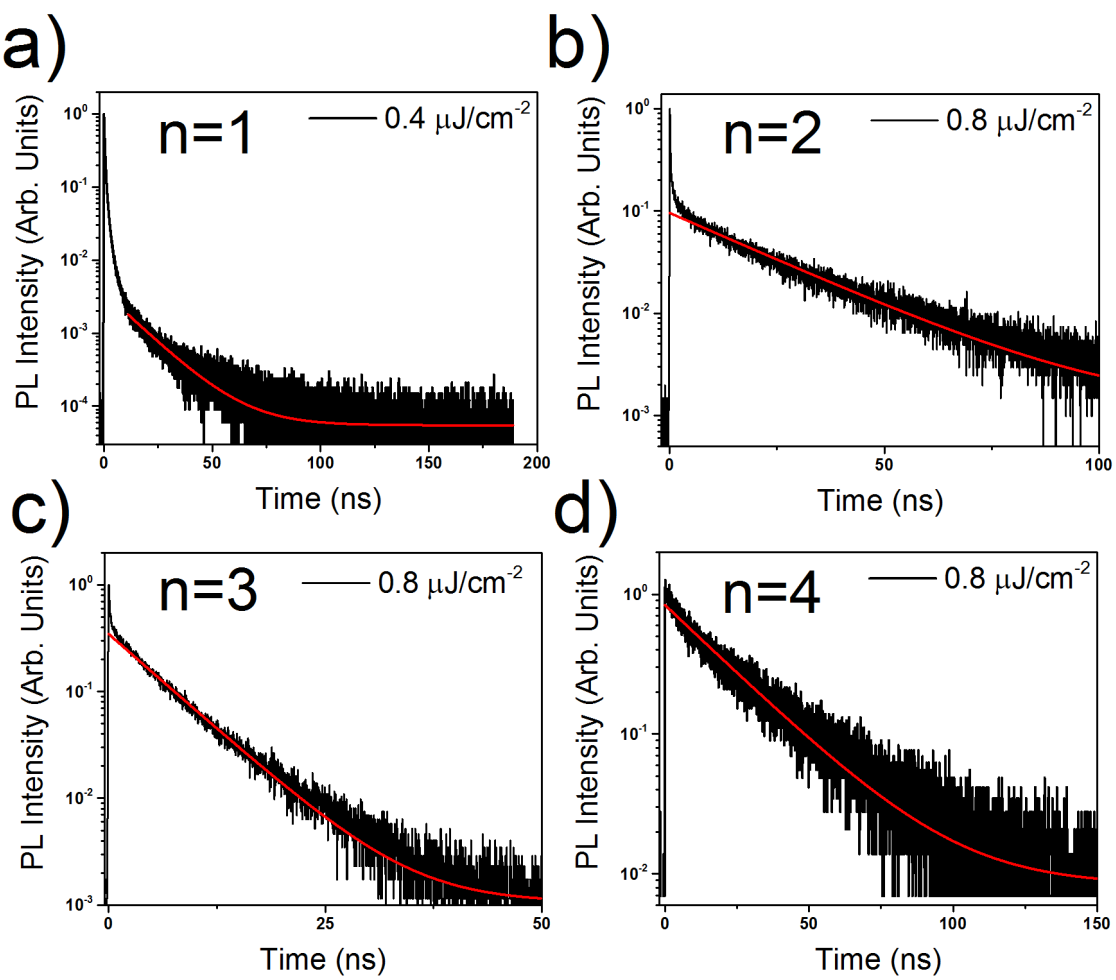

Figure S6: Mono-exponential fit of the TRPL slow component corresponding to the exciton recombination leading to lifetimes of a) $\tau=15 \pm 1$ ns for $n=1$ b) $\tau=24 \pm 1$ ns for $n=2$

c) $\tau=6.1 \pm 0.2$ ns for $n=3$ and d) $\tau=23 \pm 1$ ns for $n=4$

\section{D mass action law}

The 2D mass action law is used to describe the evolution of the free carrier fraction on the total photoexcited species, as function of the total carrier density, based on the assumption of a quasiequilibrium between excitons and free carriers:

$$
\frac{x^{2}}{1-x}=\frac{1}{n} \frac{2 \pi \mu k_{B} T}{h^{2}} e^{-\frac{E b}{k_{B} T}}
$$

$x$ is the fraction of free charge $n_{\mathrm{fc}} / n$ and $n$ is the total density of excitation $n=n_{\mathrm{fc}}+n_{x} \cdot \mu$ is the reduced mass of the exciton (approximated to $0.20 \mathrm{me}_{\mathrm{e}}$ ). ${ }^{4}$ The results are displayed on Figure $\mathrm{S7}$ for $\mathrm{n}=1,2,3$ and 4 .

The crossover density for which the free carriers and exciton density is equal $\left(n_{\mathrm{fc}}=\mathrm{n}_{\mathrm{x}}=\mathrm{n}_{\mathrm{eq}}\right)$ is given by the following equation:

$$
n_{e q}=\left(\frac{\mu_{x} k_{B} T}{2 \pi \hbar}\right) e^{-E_{b} / k_{B} T}
$$

Table S1 presents the different crossover densities. 


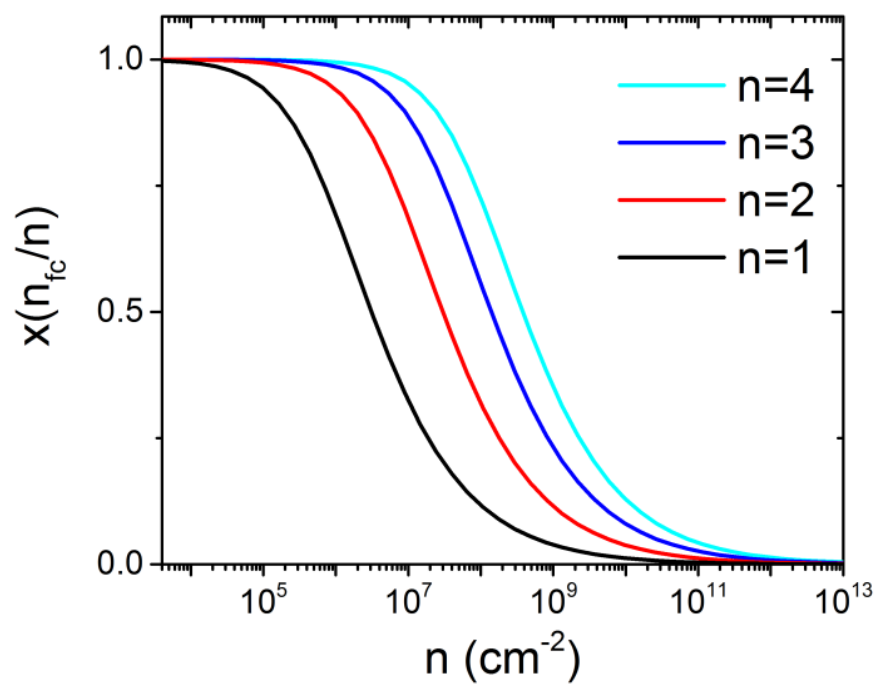

Figure S7: Free charges fractions calculated according to Saha equilibrium condition as function of the density for $\left(\mathrm{C}_{6} \mathrm{H}_{5} \mathrm{C}_{2} \mathrm{H}_{4} \mathrm{NH}_{3}\right)_{2}\left(\mathrm{CH}_{3} \mathrm{NH}_{3}\right)_{n-1} \mathrm{~Pb}_{n} \mathrm{l}_{3 n+1}(\mathrm{n}=1,2,3$ and 4$)$ at room temperature.

Table S1: Exciton binding energies and crossover densities in the RPPs crystals at 290K based on the 2D mass action law.

\begin{tabular}{|l|l|l|l|l|}
\hline & $\mathrm{n}=1$ & $\mathrm{n}=2$ & $\mathrm{n}=3$ & $\mathrm{n}=4$ \\
\hline $\mathrm{E}_{\mathrm{b}}(\mathrm{meV})$ & $220^{\mathrm{a}}$ & $170^{\mathrm{b}}$ & 125 & 100 \\
\hline $\mathrm{n}_{\text {eq }}\left(\mathrm{cm}^{-2}\right)$ & $3.110^{6}$ & $2.310^{7}$ & $1.410^{8}$ & $3.810^{8}$ \\
\hline
\end{tabular}

${ }^{a}$ From ref. ${ }^{6 \text { b }}$ From ref. ${ }^{5}$ 


\section{References}

(1) Lédée, F.; Trippé-Allard, G.; Diab, H.; Audebert, P.; Garrot, D.; Lauret, J.-S.; Deleporte, E. Fast Growth of Monocrystalline Thin Films of 2D Layered Hybrid Perovskite. CrystEngComm 2017, 8, 208-215.

(2) Peng, W.; Yin, J.; Ho, K. T.; Ouellette, O.; De Bastiani, M.; Murali, B.; El Tall, O.; Shen, C.; Miao, X.H.; Pan, J.; et al. Ultralow Self-Doping in Two-Dimensional Hybrid Perovskite Single Crystals. Nano Lett. 2017, 17, 4759-4767.

(3) Zhang, S.; Audebert, P.; Wei, Y.; Al Choueiry, A.; Lanty, G. G.; Brehier, A.; Galmiche, L.; Clavier, G.; Boissiere, C.; Lauret, J.-S. S.; et al. Preparations and Characterizations of Luminescent Two Dimensional Organic-Inorganic Perovskite Semiconductors. Materials. 2010, 3, 3385-3406.

(4) Blancon, J.-C.; Stier, A. V.; Tsai, H.; Nie, W.; Stoumpos, C. C.; Traoré, B.; Pedesseau, L.; Kepenekian, M.; Katsutani, F.; Noe, G. T.; et al. Scaling Law for Excitons in 2D Perovskite Quantum Wells. Nat. Commun. 2018, 9, 2254.

(5) Hong, X.; Ishihara, T.; Nurmikko, A. V. Dielectric Confinement Effect On Excitons In Pbi4-Based Layered Semiconductors. Phys. Rev. B 1992, 45, 6961-6964.

(6) Gauthron, K.; Lauret, J. S.; Doyennette, L.; Lanty, G.; Al Choueiry, A.; Zhang, S. J.; Brehier, A.; Largeau, L.; Mauguin, O.; Bloch, J.; et al. Optical Spectroscopy of Two-Dimensional Layered $\left(\mathrm{C}_{6} \mathrm{H}_{5} \mathrm{C}_{2} \mathrm{H}_{4} \mathrm{NH}_{3}\right)_{2} \mathrm{Pbl}_{4}$ Perovskite. Opt. Express 2010, 18, 5912-5919. 